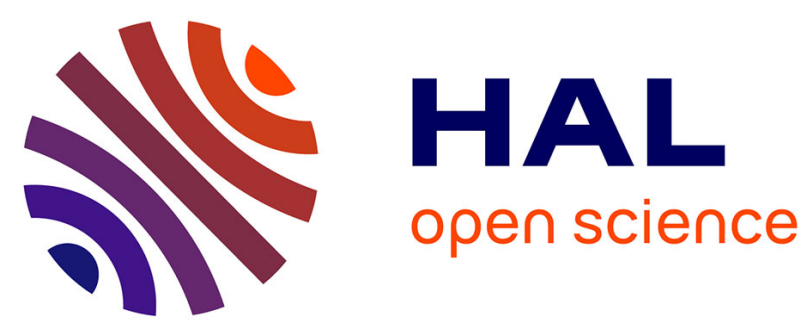

\title{
DE LA MOBILISATION À \\ L'INSTITUTIONNALISATION : UNE ANALYSE COMPARATIVE HISTORIQUE DES POLITIQUES CULTURELLES AU MEXIQUE ET EN ARGENTINE \\ Élodie Bordat-Chauvin
}

\section{To cite this version:}

Élodie Bordat-Chauvin. DE LA MOBILISATION À L'INSTITUTIONNALISATION : UNE ANALYSE COMPARATIVE HISTORIQUE DES POLITIQUES CULTURELLES AU MEXIQUE ET EN ARGENTINE. Pôle Sud - Revue de science politique de l'Europe méridionale, 2014. halshs-01712609

\section{HAL Id: halshs-01712609 \\ https://shs.hal.science/halshs-01712609}

Submitted on 22 Oct 2018

HAL is a multi-disciplinary open access archive for the deposit and dissemination of scientific research documents, whether they are published or not. The documents may come from teaching and research institutions in France or abroad, or from public or private research centers.
L'archive ouverte pluridisciplinaire HAL, est destinée au dépôt et à la diffusion de documents scientifiques de niveau recherche, publiés ou non, émanant des établissements d'enseignement et de recherche français ou étrangers, des laboratoires publics ou privés. 


\title{
«De la mobilisation à l'institutionnalisation : une analyse comparative historique des politiques culturelles au Mexique et en Argentine »
}

\section{Résumé}

\author{
Elodie Bordat-Chauvin \\ Sciences Po. Aix - CHERPA
}

L'objet de cet article est de comparer les processus et les conditions de l'institutionnalisation de politiques culturelles au Mexique et en Argentine dans les années 1980. À travers une analyse historique comparative, il étudie comment des groupes d'intérêts, d'artistes et intellectuels, se sont mobilisés pour exiger aux pouvoirs publics de se saisir du problème de la culture ; puis comment certains acteurs sont passés des «forums » de la réflexion aux arènes de la décision (Jobert, 1994), lors de l'ouverture de «fenêtres politiques » (Kingdon, 1995). Fondé sur un matériau composé d'archives et d'entretiens semidirectifs, cette contribution explore l'hypothèse selon laquelle on peut appréhender l'institutionnalisation de politiques culturelles au Mexique et en Argentine en s'intéressant à trois dimensions : les représentations des acteurs, ou «philosophies d'action» (Urfalino, 2004), présentées dans des «planifications», l'évolution du budget permettant le développement de clientèle et enfin, le cadre institutionnel de l'action publique.

\section{Mots clés}

Politiques culturelles - institutionnalisation - changement - analyse historique comparative groupes d'intérêt - Amérique latine

\begin{abstract}
This article compares cultural policies' institutionalisation processes in Mexico and Argentina in the 1980s. Through a comparative historical analysis, it studies how interest groups of artists and intellectuals mobilised in order that public authorities face the issue of culture. It then examines how some actors moved from reflexion "forums" to decision arenas (Jobert, 1994) when a political window opened (Kingdon, 1995). Based on archives and semi-structured interviews, this paper explores the hypothesis that the institutionalisation of cultural policy in Mexico and Argentina can be understood by drawing on the following three dimensions: representations, or "philosophies of actions" (Urfalino, 2004) presented in "planifications", budgetary evolution allowing a clientèle to develop, and finally, this public policy's institutional frame.
\end{abstract}

\section{Key words}

Cultural policies - institutionalisation - change - comparative historical analysis - interest groups - Latin America

Comment des politiques culturelles se sont-elles institutionnalisées au Mexique et en Argentine dans les années $1980 ?^{1}$ À travers une «analyse historique comparative » (Mahoney, Rueschemeyer, 2003), cet article propose de répondre à cette question en revenant sur des mobilisations d'intellectuels et d'artistes exigeant une réforme de l'action culturelle des pouvoirs publics. En prenant en compte la temporalité des enchaînements causaux, nous verrons si les mêmes processus et événements entraînent les mêmes effets dans les deux pays. L'approche néo-institutionnaliste adoptée ici prend ainsi en compte l'interdépendance entre les structures, les pratiques et les idées en les replaçant dans leurs contextes institutionnels et

\footnotetext{
${ }^{1}$ Je remercie Philippe Teillet et les reviewers anonymes pour leurs commentaires et suggestions.
} 
historiques. Le matériau mobilisé a été recueilli lors de quatre séjours « de terrain » entre 2008 et 2010 où un travail sur archives et une centaine d'entretiens semi-directifs ont été menés avec les principaux acteurs des politiques culturelles entre 1983 et 2009.

La politique culturelle n'est pas une «catégorie d'intervention publique transhistorique » et transposable à toute configuration institutionnelle (Dubois, 1999: 7, 8). En outre, elle ne se limite pas à la somme des parties qui la compose. Nous appellerons « actions culturelles » les diverses interventions publiques dans les secteurs des arts, des lettres et du patrimoine, et nous réserverons les termes «politique culturelle» pour «le moment de convergence et de cohérence entre, d'une part, des représentations du rôle que l'État peut faire jouer à l'art et à la 'culture' à l'égard de la société et, d'autre part, l'organisation d'une action publique » (Urfalino, $2004: 13$ ).

Le Mexique et l'Argentine possèdent assez de points communs (historique, politique et économique) et de différences (composition de la population ${ }^{2}$, types d'autoritarisme ${ }^{3}$, ampleur des crises) pour être comparables. Nous montrerons que les processus d'institutionnalisation de politiques culturelles présentent de nombreuses similarités, même si les résultats, à la fois les outputs et les outcomes (Lascoumes, Le Galès, 2011) sont différents. Le changement survenu lors de l'institutionnalisation des politiques culturelles sera appréhendé à travers plusieurs dimensions: les idées ou représentations, le cadre institutionnel et budgétaire (notamment le découpage des compétences dans les appareils politico-administratifs) et les acteurs de l'action publique. Nous prendrons en compte à la fois les facteurs exogènes de changement - contexte socio-économique et politique, crises ou changements de modèle de développement - et endogènes - capacité de l'administration, stabilité des acteurs en poste, etc.

Dans quelle mesure des mobilisations d'acteurs culturels sont-elles à l'origine de l'institutionnalisation de politiques culturelles dans les deux pays étudié ? Les politiques culturelles qui ont émergé au Mexique et en Argentine dans les années 1980 ont-elles les mêmes caractéristiques ?

\section{DES MOBILISATIONS EXIGEANT DES RÉFORMES DES POLITIQUES CULTURELLES}

L'action des pouvoirs publics dans la culture doit être située dans un contexte historique, institutionnel, politique et culturel afin de pouvoir identifier et comparer les acteurs et les configurations permettant de comprendre les processus de changement et d'institutionnalisation des politiques culturelles mexicaine et argentine. Ce sera l'objet de la première partie, alors que la seconde comparera les mobilisations d'intellectuels et d'artistes en faveur d'une transformation des actions culturelles dans les deux pays.

\section{Les premières actions culturelles argentine et mexicaine}

De l'Indépendance au premier tiers du $\mathrm{XX}^{\mathrm{e}}$ siècle, les actions culturelles argentines et mexicaines se résument à des «politiques distributives» en fournissant des services, et « régulatrices » (Lowi, 1972), en faisant adopter des normes et des lois dans les domaines des arts et du patrimoine. À partir des années 1930 des «politiques institutionnelles constitutives » (Quermonne, 1985) sont mises en œuvre et des organisations censées diriger les actions culturelles publiques sont créées. Le département chargé de la culture dépend

\footnotetext{
${ }^{2}$ La part des populations indiennes dans la population mexicaine est bien plus importante qu'en Argentine. Ce pays a pour sa part accueilli des millions d'immigrants entre la fin du XIX $\mathrm{X}^{\mathrm{e}}$ siècle et le début du $\mathrm{XX}^{\mathrm{e}}$ siècle.

3 Au Mexique le même «parti hégémonique » (Sartori, 1976) a gouverné, le pays de 1928 jusqu'en 2000 alors que l'Argentine a connu six dictatures entre 1930 et 1983.
} 
depuis lors, et jusqu'à aujourd'hui, du Secrétariat ${ }^{4}$ à l'Éducation Publique. En revanche, en Argentine, la culture dépend tour à tour de la présidence et du ministère de l'Éducation. La trajectoire et la «force institutionnelle ${ }^{5}$ (Levitsky, Murillo, 2009) des pays expliquent pourquoi l'appareil bureaucratique argentin est plus dépendant du politique que le mexicain. Ce dernier connaît des «déplacements institutionnels en série » (Ibid), c'est-à-dire que l'arrangement précédent est discrédité et les règles sont remplacées avec fréquence. Comme une partie des acteurs politiques, administratifs et intellectuels est exclue du jeu politique, dès que l'opposition prend le pouvoir, les institutions sont démantelées ou profondément modifiées. Les acteurs anticipant le fait qu'ils peuvent être remplacés ne s'investissent pas dans la création d'institutions fortes. Les coûts du changement sont abaissés et l'instabilité devient auto-renforçante. Si l'appareil politico-administratif culturel est maintenu pendant les dictatures argentines, les missions du secrétariat à la Culture sont transformées. Il ne soutient plus la culture, mais surveille étroitement les contenus culturels, et persécute de nombreux créateurs. En 1983, le pays a perdu sa place de leader continental de la production éditoriale et cinématographique et ne soutient presque plus la création culturelle. C'est dans ce contexte que la communauté artistique et intellectuelle se mobilise, pour exiger la fin de la censure et une politique culturelle démocratique. Au Mexique, le contrôle des contenus culturels et l'attribution discrétionnaire des fonds publics entraînent la mobilisation des groupes d'artistes et d'intellectuels.

\section{Analyse comparative des mobilisations collectives exigeant une transformation du traitement public de la culture}

Afin d'étudier les mobilisations des intellectuels et artistes pour la mise en œuvre de politiques culturelles en Argentine et au Mexique, il convient de revenir sur leurs positions au sein de la structure sociale et dans le champ politico-administratif et de définir ce que l'on entend par « intellectuel».

Le Mexique et l'Argentine sont les principaux exportateurs de livres du continent, jusqu'aux années 1970, mais, alors que l'État mexicain est le principal éditeur du pays, en Argentine, ce sont des politiques de soutien à la classe moyenne et l'efficacité des politiques d'alphabétisation, qui ont contribuées au développement de cette industrie. Au Mexique, nombre d'intellectuels et d'artistes mexicains vivent grâce aux commandes publiques, et à des postes dans l'administration (diplomates, responsables culturels, etc.). En revanche, le secteur culturel argentin est beaucoup plus indépendant vis-à-vis des pouvoirs publics. De nombreuses associations professionnelles et fondations, attribuent des prix et des bourses encourageant la création et la diffusion de la culture, et le secteur florissant de l'édition et de la presse permet aux intellectuels de vivre de leur plume. L'instabilité politique, économique et institutionnelle du pays et les «purges » dans l'administration publique et dans les universités auxquelles se livre le groupe vainqueur, lors des changements de gouvernement, conduisent de nombreux artistes, intellectuels et universitaires à développer leurs activités en marge de l'État grâce au soutien de fondations. Le secteur privé non-marchand a donc un rôle central en Argentine, alors qu'au Mexique, l'État est le principal financeur de la culture.

En plus de penser, écrire et enseigner, l'intellectuel traduit et interprète la réalité dans le champ politique. Les caractéristiques du groupe social des intellectuels permettent d'expliquer pourquoi il parvient à inscrire à l'agenda public ses demandes. Au Mexique, ces acteurs sont proches des arcanes du pouvoir et forment un groupe auquel l'élite politicobureaucratique s'identifie. Nombre de Présidents et de cadres du parti ont fait de hautes

\footnotetext{
${ }^{4} \mathrm{Au}$ Mexique, on appelle « secretaría » les ministères, les sous-secrétariats correspondent au secrétariat d'État. En revanche, en Argentine, on distingue les ministères des secrétariats d'État de la même façon qu'en France.

${ }^{5}$ Dans les «environnements institutionnels faibles », le degré d'application des règles formelles est faible ou discrétionnaire.
} 
études et montrent un intérêt pour les arts et les lettres. Ils cumulent des ressources sociales (réseau de relations), scientifiques (connaissances du secteur) et politiques (accès aux élus) qu'ils mettent au service de leurs mobilisations. Leur statut de figure publique et le capital de sympathie et de respect qu'ils possèdent auprès de la société, expliquent l'écoute des pouvoirs publics dont ils bénéficient. Suite aux critiques que le Président Echeverría adresse aux artistes dans son rapport de gouvernement de 1975, un groupe d'écrivains dramaturges et artistes $^{6}$, réunis derrière Octavio Paz, publient une série d'articles et de lettres ouvertes dans le quotidien l'Excelsiór et le supplément culturel Plural dans lesquels ils critiquent l'action culturelle publique et dénoncent les mécanismes de financement discrétionnaire de la culture. Ils utilisent des modes de mobilisation caractéristiques des groupes d'intérêt : "stratégies de lobbying externe » (Saurugger, Grossman, 2012 : 83) en faisait circuler pétitions et lettres ouvertes et ils ont recours « à la morale » (Offerlé, 1998) en cherchant à scandaliser le lectorat et en dénonçant les problèmes causés par le contrôle et la censure de la culture. Par ailleurs, ils ont «recours à l'expertise» (Ibid), en montrant leur connaissance du secteur et en exposant des solutions. Ainsi, ils proposent de créer un fonds pour soutenir les arts, qui allouerait des subventions selon les décisions prises par une commission de pairs, et non pas par des fonctionnaires (Paz 1975a et b). Peu de temps après la mobilisation, le Président Echeverría fait fermer l'Excelsiór qui critique trop le pouvoir.

Les intellectuels et artistes continuent à exiger une réforme de l'action culturelle mais en utilisant des modes d'actions différents. Certains sont invités à réfléchir au programme culturel du candidat du PRI, lors de la campagne de 1982. L'élaboration du programme de M. de la Madrid est confiée à la «Commission de la Culture ». Dirigée par un écrivain, elle est chargée de prendre en compte les demandes d'intellectuels et d'artistes invités à une réunion publique. Plusieurs membres de la mobilisation de $1975^{7}$ sont présents et continuent à dénoncer le caractère discrétionnaire de l'allocation des fonds pour la culture et le manque de direction claire de l'action culturelle. Face à cette situation, l'historien et ancien député du PRI, J. L. Martinez demande la création d'une structure administrative qui serait chargé de mettre en place une véritable politique culturelle. Les acteurs mobilisés ont donc à présent recours à la «négociation » (Saurugger, Grossman, 2012) dans le sens où les dirigeants politiques les invitent à participer au processus décisionnel en encadrant institutionnellement leur participation. Ce mode de mobilisation est propre aux systèmes néo-corporatistes. Suite à ces réunions, l'Institut d'Études Politiques et Sociales (IEPES), think tank du PRI, compile et publie les différentes interventions des intellectuels et présente le programme culturel du candidat en reprenant certaines demandes (comme la création d'une organisation culturelle, l'augmentation des budgets culturels).

Pendant la campagne de 1988, C. Salinas rencontre de manière informelle, A. Chumacero et V. Leñero, A. Azuela et J. L. Martinez, intellectuels et artistes qui avaient participé aux deux mobilisations précédentes, afin de recueillir leurs demandes. Si les intellectuels se mobilisent ici avec le mode de la «consultation » (Saurugger, Grossman, 2012), ils ne laissent pas de côté la «négociation» et participent au forum officiel. La commission "Société et culture » réunit des artistes (V. Flores Olea), des universitaires (G. Estrada) et des diplomates ( $R$. Tovar) et est chargée de recueillir les demandes d'artistes et d'intellectuels conviés aux réunions publiques afin de rédiger le programme culturel du candidat. On peut qualifier cette commission de «forum de communauté de politique publique » puisque les acteurs qu'elle réunit cherchent à conseiller les dirigeants et à fabriquer

\footnotetext{
${ }^{6}$ S. Elizondo, J. E. Pacheco, J. Rulfo, J. Sabines, T. Segovia, E. Carballido, J. de la Colina, S. Elizondo, J. Ibargüengoitia, C. Monsivaís, M. A. Montes de Oca, E. Poniatowska, C. Pellicer, J. Revueltas.

${ }^{7}$ Le politiste G. García Cantú et le poète M. A. Montes de Oca.
} 
des «recettes » et programmes (Jobert, 1994). Lors de l'une des réunions de la commission, plusieurs membre de la mobilisation de 1975, exigent au candidat de supprimer la censure et de «créer un Fonds national des Arts » (Salinas de Gortari, 1988 : 32). Cette demande est reprise par le candidat dans son programme qui cite presque textuellement les 8 et 12 points de la proposition d'Octavio Paz (Salinas de Gortari, 1988 : 23 ; Paz, 1975).

En Argentine aussi des propositions d'intellectuels et d'artistes pour réformer l'action culturelle nationale sont reprises par un candidat en campagne. Toutefois, la mobilisation est différente. Cela s'explique notamment par la position de ces acteurs dans la structure sociale. Lorsque l'activité politique est de nouveau autorisée en 1983, un groupe ${ }^{8}$ à la tête duquel se trouve le dramaturge Carlos Gorostiza, visite tous les candidats à l'élection. Il leur demande de prendre des engagements vis-à-vis de la culture, et notamment d'abolir la censure et de rétablir la liberté de création (propos recueillis le 17-05-2010 avec M. A., et le 18-05-2010 avec C. G.). Ce groupe possède des ressources sociales de par leurs métiers et trajectoires (plusieurs ont pris part à un célèbre mouvement artistique contestataire ${ }^{9}$ ) et dispose de ressources politiques suffisamment importantes pour leur permettre d'avoir accès aux candidats aux présidentielles. Comme au Mexique, ce groupe critique l'action culturelle et souligne l'injuste traitement des artistes apparaissant ainsi comme des re-adjusters (Cobb, Elder 1983). Le groupe se rapproche ensuite de R. Alfonsín et de son parti et participe à la création d'un espace de débat : le Centre de Participation Politique (CPP). Invités à participer au processus de réflexion sur l'action publique, les artistes, dramaturges et écrivains utilisent donc également le répertoire de la «négociation ». Toutefois, le forum officiel est différent du mexicain. L'atelier «Culture et médias » du CPP regroupe une centaine de professionnels de la culture souhaitant établir un diagnostic et proposer des solutions pour refonder l'action culturelle. Ses membres utilisent leurs « ressources scientifiques » pour se mobiliser, ou plutôt représenter leurs intérêts sous forme d'expertise. En effet, certaines demandes sont formulées de telle manière qu'elles pourraient être adoptées en l'état, sous forme de décrets. Elles se regroupent en trois catégories :

- création de cadres institutionnels formels (loi sur le théâtre, le livre,...) ;

- modification d'instruments (suppression de la censure, prélèvement de $1 \%$ sur les gains des jeux de hasard pour financer des orchestres);

- et création d'organisations (Institut du livre, ministère de la Culture...).

La commission peut donc être à la fois appréhendée comme un «forum des experts » (regroupant différents spécialistes sectoriels) mais aussi un «forum de la société civile» (englobant «des acteurs de la société civile [...] qui peuvent se mobiliser autour d'une politique publique » (Boussaguet, Muller, $2005: 34$ ) et partagent leurs expériences).

Suite à ces ateliers, un rapport est rendu au candidat et sert de base à la rédaction de la plateforme culturelle d'Alfonsín » (propos recueillis le 31-05-2010 avec L. G.). Grâce à la comparaison du programme et du livre du CPP nous avons pu voir que certaines définitions sont mot pour mot identiques ${ }^{10}$, et que les principaux objectifs de la politique (protection des industries culturelles, nécessité de démocratiser la culture sont les mêmes (Alfonsín, 1983 : 74) ; CPP, 1983 : 7) sont les mêmes. Notons enfin que les lois demandées par la commission du CPP ont été présentées au Congrès.

\footnotetext{
${ }^{8}$ Comprenant les acteurs A. Iglesias, A. Boero et M. Strat et le metteur en scènes O. Bonet.

${ }^{9}$ Teatro Abierto réunit dès 1981 des professionnels du théâtre et critique la répression à travers des métaphores.

${ }^{10}$ «Une politique culturelle cohérente ne peut se tracer sans une [...] interaction avec [...] : l'éducation, les avancées scientifiques et technologiques et les médias de masse » (CPP, 1984 : 9), (Alfonsín, 1984 :75). «La culture est une dimension fondamentale du processus de développement et de croissance d'un pays et contribue à renforcer son indépendance et sa souveraineté », (CPP, 1984 : 7 ; Alfonsín 1984 : 74).
} 
Le tableau suivant compare les mobilisations d'intellectuels dans les deux pays.

Comparaison des premières mobilisations pour l'émergence de la culture sur l'agenda public

\begin{tabular}{|c|c|c|}
\hline & Mexique, 1975, 1982, 1988 & Argentine, 1983 \\
\hline Contextes & $\begin{array}{l}\text { Réponses aux critiques du Président, } \\
\text { campagnes }\end{array}$ & $\begin{array}{l}\text { Fin de la proscription des activités } \\
\text { politiques }\end{array}$ \\
\hline Ressources & $\begin{array}{l}\text { Sociale } \\
\text { Sociétale } \\
\text { Politiques } \\
\text { Scientifiques }\end{array}$ & $\begin{array}{l}\text { Sociale } \\
\text { Sociétale } \\
\text { Politiques } \\
\text { Scientifiques }\end{array}$ \\
\hline $\begin{array}{c}\text { Mode de } \\
\text { mobilisation }\end{array}$ & $\begin{array}{l}\text { Lobbying externe, } \\
\text { Recours au nombre, à la morale, à la } \\
\text { négociation et à la consultation }\end{array}$ & $\begin{array}{l}\text { Lobbying interne, } \\
\text { Recours à la morale, à l'expertise, et à la } \\
\text { négociation }\end{array}$ \\
\hline Forums & $\begin{array}{l}\text { Forum de communauté de politique } \\
\text { publique }\end{array}$ & $\begin{array}{l}\text { forum des experts } \\
\text { forum de la société civile }\end{array}$ \\
\hline
\end{tabular}

\section{Élaboration propre.}

L'une des principales différences entre les deux mobilisations, réside dans leur temporalité. La mexicaine commence en 1975 et est reprise ponctuellement pendant les campagnes avec un recours à la consultation. La mobilisation argentine commence à la fin de la dictature et a recours à l'expertise. Dans les deux cas, les acteurs ont également recours à la morale et à la négociation et exigent la fin de la censure et la mise en place de mécanismes soutenant la culture. Nous avons vu que la comparaison des propositions des intellectuels dans les forums et des promesses de campagne montre que leurs demandes ont été reprises par les candidats, mais dans quelle mesure ces promesses ont-elles été traduites en actions publiques?

\section{L'ouverture d'une fenêtre politique et le passage des forums aux arènes}

Nous mettrons ici en lumière les points communs entre les processus d'ouverture de fenêtres politiques ayant permis l'inscription de la culture sur l'agenda public dans les deux cas ; puis nous soulignerons que les recettes proposées dans les forums sont prises en compte au moment où les leaders de ces groupes accèdent à des postes de responsabilité.

Une fenêtre politique s'ouvre lors de la confluence de trois courants indépendants (Kingdon, 1983). Le courant des problèmes regroupe le manque de financements publics, la censure et les actions culturelles erratiques des pouvoirs publics. Le courant des solutions regroupe la création d'institutions, d'instruments économiques, la modification de cadres institutionnels, etc. Le couplage des solutions avec les problèmes (par exemple l'élimination de la censure en Argentine en supprimant 1'Entité de Qualification) et le moment où ces courants rejoignent le courant politique, dépendent du talent et des ressources d'entrepreneurs politiques. Le courant politique est activé en Argentine par l'élection d'Alfonsín et un changement de régime, alors qu'au Mexique il s'agit de l'élection de Salinas et de la perte de la majorité du PRI à la chambre basse. Les conditions de l'élection de ce dernier ${ }^{11}$ l'entraînent à chercher à regrouper le maximum de soutiens au début de son mandat. Le tableau suivant synthétise ces éléments.

\footnotetext{
${ }^{11}$ Alors que le candidat de l'opposition est en tête, un «problème informatique » survient. Salinas est désigné vainqueur, mais son élection est entachée par les suspicions de fraude.
} 


\section{Comparaison de l'ouverture des fenêtres politiques dans les deux pays}

\begin{tabular}{|c|l|l|}
\hline & \multicolumn{1}{|c|}{ Argentine } & \multicolumn{1}{c|}{ Mexique } \\
\hline $\begin{array}{c}\text { Problem } \\
\text { stream }\end{array}$ & $\begin{array}{l}\text { - censure, } \\
\text { - manque de financements publics, } \\
\text { - crise économique. }\end{array}$ & $\begin{array}{l}\text { - censure, } \\
\text { - financements discrétionnaires, } \\
\text { - achats de volontés, clientélisme, } \\
\text { - actions culturelles éparses, désorganisées. }\end{array}$ \\
\hline $\begin{array}{c}\text { Policy } \\
\text { stream }\end{array}$ & $\begin{array}{l}\text { - dissolution de l'Entité de qualification, } \\
\text { - mécanismes de soutien à la création } \\
\text { - création d'un ministère de la Culture. }\end{array}$ & $\begin{array}{l}\text { - création d'un fonds pour la culture et } \\
\text { d'une organisation dirigeant le secteur. }\end{array}$ \\
\hline $\begin{array}{c}\text { Political } \\
\text { stream }\end{array}$ & $\begin{array}{l}\text { - élection de R. Alfonsín } \\
\text { - passage d'une dictature militaire à un } \\
\text { régime présidentiel. }\end{array}$ & $\begin{array}{l}\text { - élection de C. Salinas } \\
\text { - le PRI perd la majorité absolue à la } \\
\text { Chambre basse. }\end{array}$ \\
\hline
\end{tabular}

\section{Élaboration propre.}

C. Salinas doit gouverner avec le stigmate de l'illégitimité, il prend alors des décisions lui permettant de réaffirmer son statut de Président. La création du Conseil national pour la Culture et les Arts (CONACULTA), une semaine après sa prise de fonction, et du Fonds National pour la Culture et les Arts (FONCA) par décret présidentiel, ne doit pas uniquement être comprise comme la réponse à une demande de secteurs sociaux influents, mais comme « l'acte politique » d'un Président en quête de soutien politique (propos recueillis le 26-112009 avec G. E, et le 20-11-2009 avec J. R. D). D'autres éléments permettent d'inscrire ces créations dans le cadre d'échanges clientélaires ${ }^{12}$. Au début de son mandat, le Président fait arrêter de manière spectaculaire un puissant leader syndical, surnommé «la Quina». Quelques jours plus tard, deux télégrammes signés par des intellectuels et artistes sont envoyés à la presse et à la résidence présidentielle pour assurer Salinas de leur «soutien inconditionnel ». Des signataires souhaitant rester anonymes nous racontent :

«Depuis les bureaux du CONACULTA, on a cherché à faire signer ce document [...] par des intellectuels [...]. C'était le prix à payer imposé au Conseil pour sa survie... C'est-à-dire la protection même du secteur ».

Il s'agit donc ici «de promesses de loyauté et de solidarité » (Roniger, $1990: 3$ ) faites par les intellectuels au Président en échange de l'aboutissement de leurs demandes. Au fil du mandat de Salinas, le FONCA s'est transformé en « un vaste programme de bourses [...] dont l'objectif était, si ce n'est contrôler les intellectuels, au moins d'avoir leur sympathie » (Volpi, 2000). Lorsque ces derniers demandent en 1992 la création d'une chaîne culturelle dans le cadre de la privatisation de chaînes publiques, Canal 22 est créée et son administration leur est confiée. En retour, les intellectuels médiatiques continuent à soutenir publiquement le Président.

C. Salinas et R. Alfonsín nomment les membres des forums les plus dotés en ressources sociales et sociétales à la tête de leur politique culturelle. Ils nomment à leur tour des membres des forums à des postes clés. Carlos Gorostiza ${ }^{13}$ est nommé en Argentine et le

\footnotetext{
${ }^{12}$ Le clientélisme peut être défini comme «la distribution (ou la promesse) de ressources par des hommes politiques, candidats ou en poste, en échange d'un soutien politique » (Auyero, $2010: 74$ ).

${ }^{13}$ Membre fondateur de Teatro Abierto, Prix national de Littérature (1978), et du Konex de Platine (1984). Il est instigateur de la première mobilisation des artistes et un entrepreneur politique qui a œuvré au couplage des solutions et des problèmes. Il nomme M. Aguinis (écrivain) sous-secrétaire, P. O’Donnell (psychanalyste) secrétaire à la Culture de Buenos Aires, M. Antín (cinéaste) directeur de l'Institut du Cinéma, cinq autres membres du CPP sont nommés à des directions nationales et autres postes dans l'organisation culturelle.
} 
Víctor Flores Olea ${ }^{14}$ au Mexique. Ils publient des documents présentant leur politique culturelle reprenant les grands objectifs présentés dans les forums : abolition de la censure, démocratisation culturelle, défense de l'identité culturelle nationale, décentralisation, adoption d'instruments encourageant la création et son financement (Secretaría de Cultura, 1984, CONACULTA, 1990). Toutefois, si les membres du CPP inscrivaient leurs demandes dans l'objectif central de la «démocratie culturelle » (où tous les citoyens sont considérés comme des créateurs), les objectifs des directions nationales du Plan National de la Cutlure ${ }^{15}$ nous font davantage inscrire son action dans la démocratisation de la culture (c'est-à-dire rendre accessibles aux citoyens les œuvres de la « haute culture ») ${ }^{16}$. En revanche, dans le Programme mexicain, l'objectif central est le même que celui proposé dans le forum, et s'inscrit dans le modèle de la « démocratie culturelle ${ }^{17}$.

Le passage de certains acteurs des forums aux arènes a été possible grâce à des facteurs similaires : l'ouverture d'une fenêtre politique, intérêt du nouveau Président pour la culture, et la volonté d'intellectuels et d'artistes de travailler dans l'administration publique. Les principaux acteurs de l'institutionnalisation de la politique culturelle mexicaine et argentine ont ainsi pensé la politique culturelle depuis les forums, avant de la mettre en œuvre depuis l'administration culturelle, les «arènes » ${ }^{18}$. Dans quelle mesure peut-on parler de l'institutionnalisation de politiques culturelles dans les années 1980 en Argentine et au Mexique et quels éléments permettent d'appréhender ce processus ?

\section{LES PROCESSUS D'INSTITUTIONNALISATION ET DE CHANGEMENT DES POLITIQUES CULTURELLES ARGENTINE ET MEXICAINE}

Synonyme de «reconnaissance officielle (Lenoir, 1995) ou de « routinisation des pratiques » (Dulong, 2012), l'institutionnalisation est un processus. En France, l'institutionnalisation d'une politique culturelle survient à la suite de la création du ministère des Affaires culturelles, lorsque les opérateurs de l'objectivation «produite et donnée à voir dans des institutions [...] des rôles politiques et administratifs [...] des discours et des textes [...] » sont réunis (Dubois, 1999:148). La planification revient à la production d'une «doctrine d'action culturelle » (Dubois, 1999: 153) et contribue à la légitimation de la politique, tout comme la consolidation d'un cadre normatif et l'augmentation progressive du budget. Sur nos terrains, appréhender la manière dont les acteurs intériorisent et se réapproprient les façons de penser et d'agir est difficilement repérable. Nous avançons que l'institutionnalisation des politiques culturelles peut être appréhendée en s'attachant aux cadres institutionnels garantissant la pérennité de la politique au-delà des changements politiques, aux budgets permettant de financer l'action publique et de constituer une clientèle,

\footnotetext{
${ }^{14}$ Photographe, directeur de la faculté de sciences politiques de l'UNAM (1970-75), ambassadeur du Mexique auprès de l'UNESCO (1978-82) il est sous-secrétaire à la Culture (1977-78). Il nomme trois membres de la Commission « Culture et société » à des postes de responsabilité.

15 «Préserver le patrimoine de la nation », « démocratiser l'accès aux musées », « soutenir le sauvetage musical des communautés autochtones » (Secretaría de Cultura, 1984). Les missions du secrétariat sont : la liberté de création, l'encouragement à la production culturelle, la participation à la distribution des biens et services culturels et la préservation du patrimoine culturel de la nation (Ibid), ce qui rappelle celles de Malraux.

${ }^{16}$ Selon cette conception l'État doit d'assurer à tous la possibilité de participer à la vie culturelle. Elle est remplacée dans les années 1970 par la « démocratie culturelle ».

${ }^{17}$ Le rôle du Conseil est de «susciter une participation ample [...] des Mexicains dans la politique culturelle » (CONACULTA, $1990:$ 19).

${ }^{18}$ Il s'agit d'espaces dans lesquels se négocient les compromis institutionnalisés et se produisent les politiques publiques et leur révision (Jobert, 1994).
} 
et enfin, à la présence d'une «ressaisie des idées et des initiatives » (Urfalino, $2004: 360$ ), c'est-à-dire une «philosophie d'action », formalisée et diffusée dans des documents.

\section{Des cadres institutionnel et budgétaire plus ou moins consolidés}

Le cadre institutionnel prévoit et prescrit les comportements des acteurs individuels et collectifs. Plus ce cadre est fort et stable, plus l'abandon d'une action publique est difficile. Les organisations fournissent des normes, des pratiques et des représentations qui se consolident au fil du temps. Lorsque leurs membres développent un sentiment d'appartenance et partagent des valeurs et croyances propres, ils tendront à défendre leur organisation. En outre, la mise en œuvre de politiques redistributives, permet de développer des clientèles qui défendront également l'institution et la politique (Pierson, 1993).

La politique culturelle est formulée depuis le CONACULTA au Mexique et le secrétariat argentin à la Culture. Ce sont les lieux où le dialogue avec les parties prenantes se déroule et à partir duquel l'action est implémentée. Quelles sont les similitudes et les divergences entre les modèles d'organisation choisis par les deux pays ? Le CONACULTA est chargé des missions culturelles revenant jusqu'alors au SEP, de réorganiser l'ensemble du secteur culturel public et de formuler et d'exécuter une politique nationale. Plusieurs structures administratives passent sous sa direction. Il s'agit d'un changement par « déplacement », où l'arrangement institutionnel traditionnel est discrédité et remplacé par une nouvelle organisation associée à de nouveaux comportements (Streeck, Thelen, 2005 : 20). Ce déplacement provoque de nombreux conflits puisqu'anciennes et nouvelles règles cohabitent. Tout comme les organismes qu'il est chargé de diriger, le Conseil est un organisme déconcentré. Or s'il est créé par décret, l'INBA et l'INAH ont des lois de création, c'est pourquoi ces organisations considèrent le Conseil illégitime pour les diriger et mener la politique culturelle.

Les transformations du secteur culturel mexicain à la fin des années 1980 sont importantes, car aux facteurs endogènes s'ajoutent un facteur exogène : le changement de modèle de développement. Les recettes keynésiennes sont remplacées par des monétaristes et la réduction de l'État se traduit par le démantèlement de mécanismes de soutien ; alors que l'ouverture économique se traduit par la négociation de l'Accord de Libre-Échange Nord Américain. Toutefois, les budgets de la culture augmentent avec C. Salinas : de $109 \%$ entre 1987 et 1988 (Zedillo, $1998: 214$ ), ils passent de 0,64\% des dépenses de l'État en 1989 et à $0,80 \%$ en 1992 (Zedillo, 2000 : 254). Le FONCA, à travers son programme de «créateurs d'arts » alloue des bourses de trois ans aux jeunes artistes et des bourses ad vitam aux créateurs «émérites ». Pratiquement tous les membres de la mobilisation de 1975 sont dans la seconde catégorie (CONACULTA-FONCA, 2006).

À la différence du cadre institutionnel de la culture au Mexique, l'argentin est «né faible » (Levistky, Murillo, 2011). Les interruptions de l'ordre institutionnel et les purges qui les suivent, expliquent les nombreux changements dans le niveau hiérarchique et dans l'appartenance organisationnelle du secrétariat à la Culture. Dans un contexte où l'instabilité institutionnelle est grande, ni les décideurs, ni les administrateurs, ni les professionnels de la culture ne s'engagent à consolider ce cadre, car ils anticipent le fait qu'un nouveau renversement de pouvoir peut les déplacer. L'instabilité devient alors auto-renforçante, les coûts du changement s'abaissent. Il semblerait que, malgré le retour à la démocratie, le cycle de l'instabilité institutionnelle n'ait pas été totalement enrayé. Le ministère, que les membres du CPP demandaient, ne peut être créé, la Constitution limitant le nombre de ministères. Toutefois, le secrétariat à la Culture et le Fonds National des Arts (FNA) sont totalement 
réorganisés et le FNA récupère les sources de financement dont il avait été spolié. Le secrétariat ne dépend plus de la Présidence, mais du ministère de l'Éducation (Loi 23.023 du 8-12-1983), son cadre institutionnel, et donc ses missions, sont transformés. Il s'agit ici d'un changement par «conversion institutionnelle » où l'institution reste la même, mais ses objectifs différents. Elle ne censure plus, mais encourage la création culturelle. Ces changements sont graduels, car tous les agents ayant travaillé pour la dictature n'ont pu être remplacés, parce qu'ils représentent une partie importante du personnel, et parce qu'il n'y a pas personnel plus qualifié en l'absence de formation dans la gestion culturelle. Un autre changement institutionnel survient en 1985 lorsque le secrétaire à la Culture est invité à siéger aux réunions du Cabinet (décret 12.30), puis deux ans plus tard, lorsque le secteur de la culture absorbe celui de la communication (décret $\mathrm{n}^{\circ} 84$ ). Ce changement, ayant eu lieu suite à des conflits entre les factions de l'UCR, il entraîne des remaniements ministériels et le remplacement des artistes par des gestionnaires. Le secteur de la communication devient alors plus important que celui de la culture dans les discours et les budgets.

Comme au Mexique, les transformations de la politique culturelle sont liées à des facteurs endogènes mais aussi exogènes. Les recettes monétaristes sont remplacées par des recettes néo-keynésiennes et les financements publics augmentent. Entre 1982 et 1988, le budget du secrétariat passe de $0,1 \%$ des dépenses de l'État à $0,39 \%$ (Ministerio de Economía, 2006). Les bourses allouées par le FNA ne peuvent être comparées à celles du Mexique ni dans les montants ni dans les durées. Ses fonds sont beaucoup plus limités, ils ne permettent donc pas de consolider de clientèle. De même, les nombreux remplacements des agents dans la politique culturelle ne favorisent pas cette consolidation.

Si les deux politiques ont en commun la procédure de nomination du titulaire de l'organisation par l'Exécutif, le niveau administratif et légal de l'organisation centrale et la dépendance au secteur de l'éducation, les mécanismes de financement ${ }^{19}$, les liens clientélaires liant le Président à certains intellectuels et la stabilité des agents en poste, les distinguent. En effet, on peut en partie expliquer la stabilité de la politique culturelle mexicaine par le fait que le Président Salinas et des intellectuels médiatiques continuent à échanger des services. Si dans les deux pays, le remplacement du responsable de la politique entraîne des changements dans la quasi-totalité des services, au Mexique il n'y a qu'un changement pendant le sexennat de Salinas alors qu'il y en a trois pendant celui d'Alfonsín. On observe dans les deux cas, la présence d'une institution spécifiquement dédiée à mener la politique culturelle et une augmentation de son budget mais, qu'en est-il de la « ressaisie » des idées et recette ?

\section{Des «philosophies d'action " présentées dans des planifications culturelles}

Au-delà d'une énumération d'objectifs et de bonnes intentions, le «Plan national de la Culture 1984-89» argentin et le «Programme national de la Culture 1990-94» mexicain permettent de comprendre comment la politique est pensée, construite, et donnée à voir. Ils permettent d'appréhender les représentations sur lesquelles reposent la politique, mais aussi son contenu (programmes, définitions et instruments qui vont encadrer la mise en œuvre). La présence de «philosophies d'actions » montre que la politique va au-delà de la volonté des décideurs en poste, mais qu'elle prend en compte les réflexions émanant du secteur sur lequel elle propose d'agir. Ainsi, lorsque des membres des forums sont passés dans les arènes, en étant nommés dans les instances de décision, ils ont repris certaines propositions élaborées dans ces espaces pour construire leurs planifications : au Mexique, la création du FONCA et

\footnotetext{
${ }^{19}$ Le FNA et le FONCA ont des missions et un fonctionnement différents. Le premier fonctionne comme une banque, en revanche, le rôle de banque du FONCA n'est que l'une de ses activités puisqu'il gère aussi l'attribution de bourses. Dans les deux cas, les décisions sont prises par des commissions de pairs.
} 
du CONACULTA, en Argentine, la suppression de l'Entité de Qualification et la présentation de lois sur les industries culturelles.

La politique culturelle mexicaine a comme principaux objectifs de développer et diffuser la culture, de protéger le patrimoine, et d'encourager la participation de la société. L'exécutif est chargé de renforcer l'identité nationale, de promouvoir et respecter la liberté de création et de garantir l'accès aux biens et services culturels (CONACULTA, 1990). Le secrétariat argentin à la culture se donne pour sa part quatre objectifs : « la liberté de création, l'encouragement de la production culturelle, la participation à la distribution des biens et services culturels et la préservation du patrimoine » (Secretaría de Cultura, 1984). En plus de ces objectifs, les deux politiques se voient confier de grandes missions, comme consolider l'identité nationale et participer à la décentralisation. Les définitions de la politique culturelle présentées s'inspirent de celle de l'UNESCO, adoptée lors de la Conférence mondiale des Politiques culturelles (Mexico, 1982). On y observe la même tension entre les philosophies d'action de la «démocratisation culturelle ${ }^{20}$ et à la «démocratie culturelle ${ }^{21}$ (Bordat 2013a, 2013b).

L'analyse du Plan argentin et du Programme mexicain de la Culture montre que la politique culturelle est pensée. Il y a des définitions, des missions et des objectifs définis ainsi que des instruments (fonds pour la culture) et des cadres institutionnels (lois pour les atteindre, et donc une «rationalisation de l'incertitude»(Dubois, 1999)). On peut alors affirmer que les deux pays ont mis en œuvre des politiques culturelles.

Nous avons formulé ici l'hypothèse selon laquelle l'institutionnalisation de politiques culturelles au Mexique et en Argentine est le fruit de la mobilisation de groupes d'artistes et d'intellectuels, qui, après avoir pensé les politiques depuis les forums de réflexion, ont pu mettre en œuvre leurs idées en accédant aux arènes de la décision, à la faveur de l'ouverture de fenêtres politiques. C'est la convergence de plusieurs facteurs qui a permis l'institutionnalisation de ces politiques: la consolidation d'un cadre institutionnel et budgétaire et l'adoption d'une planification prenant en compte les propositions de forums du secteur. Cette convergence a lieu dans des contextes politico-institutionnels différents. L'Argentine connaît une grave crise économique et politique à la fin de la décennie, mais les soulèvements militaires ont cessé. Dans les années 1990, le Mexique connaît à la fois un renforcement du présidentialisme et de l'autoritarisme et une progressive ouverture du jeu politique. Grâce à un budget conséquent, les organisations mexicaines ont vu se consolider autour d'elles une clientèle possédant des ressources importantes lui permettant de défendre la politique. En revanche, sur la période analysée ici, la politique culturelle argentine semble plus faiblement institutionnalisée. Le secrétariat à la Culture connaît toujours une instabilité institutionnelle, ses agents ne restent pas en poste plus de deux ans, et, malgré une augmentation, les budgets culturels continuent à être limités. Toutefois, au regard de l'évolution de la politique culturelle dans les années 1990 et 2000, c'est avec Alfonsín que la culture a eu le rang hiérarchique et le plus budget le plus important. Par ailleurs, l'abolition de la censure, la garantie de la liberté de création et la récupération de l'autonomie du FNA ont eu des conséquences majeures dans la société argentine avec l'essor d'expressions culturelles

\footnotetext{
${ }^{20}$ L'une des lignes directrices de la politique culturelle argentine est que « l'action culturelle doit promouvoir l'élévation culturelle du peuple» (Secretaría de Cultura, 1984). En outre, les programmes du secrétariat sont principalement de diffuser la danse, la musique, le théâtre. La philosophie d'action de la démocratisation culturelle l'emporte donc sur la démocratie culturelle.

${ }^{21}$ « Il faut rompre le critère élitiste selon lequel les créateurs de culture sont les artistes, et la communauté une consommatrice passive », ou encore qu'il faut faire « la promotion de la participation active des citoyens dans la construction et le développement de leur culture »(CPP, $1984: 26 ; 107)$.
} 
dynamiques, ce qui confirme l'hypothèse d'une institutionnalisation de la politique. On peut donc conclure que, malgré un degré plus faible d'institutionnalisation qu'au Mexique (qui a des budgets plus importants garantis par des échanges clientélaires entre le secteur culturel et le pouvoir, ainsi que des institutions plus stables), la politique culturelle argentine s'est institutionnalisée.

\section{BIBLIOGRAPHIE}

- Anderson B., (2002), L'imaginaire national. Réflexion sur l'origine et l'essor du nationalisme, Paris, La découverte.

- Auyero J., (2010), «Contestation et patronage : intersections et interactions au microscope », Revue internationale de politique comparée, 2010-2, vol. 17, pp. 71-102.

- Bordat E. (2013a) «Institutionalization and change in cultural policy : CONACULTA and cultural policy in Mexico (1988-2006) », International Journal of Cultural Policy, vol. 19, $\mathrm{n}^{\circ} 2$, March, p.222-248.

- Bordat E. (2013b) «"Desarrollo" y "diversidad cultural" en el marco de la integración regional y la globalización: el caso de las políticas argentinas y mexicanas » dans País Andrade M., Molina Roldán A., Cultura y desarrollo en américa latina.,Tucumán (Argentine), ediciones cooperativas, pp. 31-60.

- Cobb R., Elder C. (1983), Participation in American Politics. The Dynamics of AgendaBuilding, Maryland, The John Hopkins University Press.

- Dezalay Y., Garth B. (2002), La mondialisation des guerres de palais, Paris, Le Seuil.

- Dubois V. (1999), La politique culturelle, Genèse d'une catégorie d'intervention publique, Paris, Belin.

- Dulong D. (2012), Sociologie des institutions politiques, Paris, La Découverte.

- Jobert B. (1994), Le tournant néo-libéral en Europe, Paris, l’Harmattan, 1994.

- Lascoumes P., Le Galès P. (2011), Sociologie de l'action publique, Paris, Armando Colin.

- Levitsky S., Murillo M. V., (2009), «Variation in Institutional Strength», Annual Review of Political Science, vol. 12, 2009, pp. 115-133.

- Lowi T. (1972), «Four Systems of Policy, Politics, and Choice», Public Administration Review, vol. 32, n 4, juil.- août, pp. 298-310.

- Mahoney J., Rueschemeyer D. (2003), Comparative Historical Analysis in Social Science, New York, Cambridge University Press.

- Offerlé M. (1998), Sociologie des groupes d'intérêts, Paris, Montchrestien.

- Pierson, P. (2000), «Path Dependence, Increasing Returns, and the Study of Politics», American Political Science Review, 94 (2).

- Quermonne J.-L. (1985), «Les politiques institutionnelles. Essai d'interprétation et de typologie », dans Grawitz M., Leca J., Traité de science politique, tome 4, Les politiques publiques, Paris, PUF.

- Roniger L. (1990), Hierarchy and Trust in Modern Mexico and Brazil, New York, Praeger.

- Sartori G. (1976), Parties and Party Systems: A Framework for Analysis, Volume I, New York, Cambridge University Press.

- Sartori G. (1994), «Bien comparer, mal comparer», Revue Internationale de politique comparée, vol. 1, I, pp. 19-36.

- Saurugger S., Grossman E. (2012), Les groupes d'intérêt : Action collective et stratégies de représentation, Paris, Armand Colin.

- Streeck W., Thelen K. (2005), Beyond Continuity, Oxford, Oxford University Press.

\section{Sources}

- Alfonsín R. (1983). Ahora mi propuesta política, Buenos Aires, Sudamericana-Planeta. 
- CONACULTA (1990), Programa nacional de Cultura 1990-1994, Mexico.

- CONACULTA-FONCA (2006) FONCA. 18 años de inversión en el patrimonio vivo de México, CONACULTA, Mexico.

- CPP (1983), Cultura y democracia, Buenos Aires.

- De la Madrid M., (1982), Cultura nacional, Mexico, PRI-IEPES.

- Paz, O. (1975a), «Declaración sobre la libertad del arte », Excélsior, mai.

- Paz, O. (1975b), Plural, vol V, n 1, octobre.

- Presidencia de la República $(1998,2000)$ Cuarto y Sexto Informe de Gobierno de E. Zedillo, México.

- Salinas de Gortari C., IEPES, (1988), Dialogo nacional, Mexico, PRI-IEPES.

- Secretaría de Cultura (1984), Plan Nacional de Cultura 1984-1994, Buenos Aires.

- UNESCO (1978), Conférence intergouvernementale sur les politiques culturelles en Amérique latine et dans les Caraïbes, rapport final.

- Volpi J., (2000), «El fin de la conjura, los intelectuales y el poder en México en el siglo

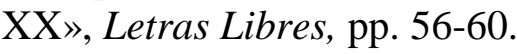

